\title{
Cross-regulation of Arabidopsis root growth by plant hormones auxins and ethylene
}

\section{Alla Korobova ${ }^{1}$, Anna Vasinskaya ${ }^{1}$, Anastasia Kirpichnikova ${ }^{2}$, Maria Shishova ${ }^{2}$, and Guzel Kudoyarova ${ }^{1}$}

${ }^{1}$ Ufa Institute of Biology, Ufa Federal Research Centre, Russian Academy of Sciences, Prospekt Oktyabrya, 69, Ufa, 450054, Russian Federation;

2Department of Plant Physiology and Biochemistry, Faculty of Biology,

Saint Petersburg State University, Universitetskaya nab. 7-9, St. Petersburg, 199034,

Russian Federation

Correspondence and requests for materials should be addressed to Alla Korobova, muksin@mail.ru

\section{Abstract}

We investigated the cross-talk between auxin (IAA) and ethylene in the control of root growth of Arabidopsis plants (Arabidopsis thaliana). The root growth of ethylene insensitive etr1-1 and auxin insensitive tir1 mutants under the effect of IAA and 1-methylcyclopropene (1-MCP, inhibitor of ethylene perception) was compared, respectively, with that of the wild type ecotype Columbia (Col-0). Roots of Col-0 were shorter than those of the etr1-1 mutant. The addition of IAA $\left(5.7 \times 10^{-6} \mathrm{M}\right)$ to the growth medium led to $20 \%$ root shortening in Col0 plants, but not in etr1-1 mutants. Thus, the capacity of plants for ethylene perception contributes to the control of root length and its sensitivity to IAA. Roots of etr1-1 were less heavy than in Col-0, indicating that ethylene maintains root mass accumulation. Treatment with IAA caused a decrease in root mass of both genotypes (resulting in a 25 and $10 \%$ decline in the root mass of Col-0 and etr11 as compared to the corresponding control), suggesting that IAA may influence root biomass accumulation independently of ethylene. However, sensitivity to ethylene increases plant responsiveness to IAA. Mutation in the auxin receptor decreased the sensitivity of roots to inactivation of ethylene receptors: treatment with 1-MCP resulted in a $40 \%$ decline in the root mass of Col- 0 and only a $10 \%$ decrease in tir1. The decrement in sensitivity to auxins in the tir1 mutant decreased the responsiveness of root biomass to 1-MCP-treatment. These data suggest an additive action of ethylene and auxins on accumulation of root biomass.

Keywords: Arabidopsis thaliana, etr1-1, tir1, ethylene, IAA, root growth, 1-MCP.

\section{Introduction}

Regulation of root growth plays an important role in plant adaptation to the environment and particularly to heterogeneous distribution of ions, which is a general feature of natural soil. When the growing roots reach patches with an increased concentration of mineral nutrients, their elongation is inhibited, which contributes to effective uptake of ions (Trapeznikov et al., 2003; Ivanov, 2009). The importance of root growth reaction determines the interest in discovering mechanisms responsible for its regulation. Ethylene and auxins are well known to inhibit root elongation (Takahashi, 2013). The similarity of plant response to either of these two hormones indicated their cross-talk in implementation of inhibitory action on root growth (Muday et al., 2012). The results of comparative transcriptomic analysis highlighted that cross-talk between ethylene and auxin is due to the mutual regulation of these hormone pools (Stepanova et al., 2007). Auxins have been shown to be capable of stimulating ethylene production (Woeste et al., 1999), while ethylene, in turn, influences the accumulation and distribution of auxins through effects on 
expression of genes coding auxin transporters (Ruzicka et al., 2007). Thus, root growth inhibition caused by ethylene and auxins is likely to be either due to ethylene-induced accumulation of auxins in the growing root zones or (alternatively) due to activation of ethylene production by auxins. Recently, preference has been given to the first of these mechanisms. Data on the absence of stimulation of ethylene production by auxin (indoleacetic acid, IAA) in isolated pea roots were obtained about 30 years ago and served as an argument against the participation of ethylene in the root growth response to auxins (Eliasson et al., 1989). Auxin-induced up-regulation of the genes responsible for ethylene synthesis was detected later (Stepanova et al., 2007) but failed to change the opinion on cross-talk between auxins and ethylene. The capacity of ethylene-insensitive Arabidopsis mutants to respond to synthetic auxin 1-naphthylacetic acid (NAA- synthetic analog of native auxins) was used as an argument against the importance of the role of ethylene in the root growth response to auxins (Ruzicka et al., 2007). Nevertheless, it is important to emphasize that auxin transporters, which are the likely targets of ethylene action (Negi et al., 2008), are less important for the transfer of NAA across membranes than that of natural auxin indoleacetic acid (Marchant et al., 1999). Thus, analysis of the data on the cross-talk between auxins and ethylene during the control of root growth is still insufficient. We planned to solve this problem by comparing IAA effects on the root growth of ethylene insensitive Arabidopsis mutant etr1-1 and its wild type ecotype Columbia (Col-0). Along with root length, we also measured root fresh weight, since the decrease in root mass of etr1-1 as compared to Col- 0 has been shown by us previously (Korobova et al., 2016), as well as the capacity of root mass accumulation to emphasize plant adaptation (Kudoyarova et al., 2015). We also compared root growth responses to the inhibitor of ethylene perception, 1-methylcyclopropene (1-MCP), in plants with mutation in the auxin receptor TIR1 to that of the wild type genotype. The aim of the present work was to reveal dependence of auxin-induced changes in root growth on their sensitivity to ethylene as well as to discover the action of the inhibitor of ethylene perception under the changes in plant sensitivity to ethylene.

\section{Materials and Methods}

Experiments were performed on the plants of Arabidopsis thaliana [L.] Heynh. Ecotype Columbia (Col-0), ethylene insensitive mutant etr1-1 and auxin insensitive mutant tir1. After stratification on wet filter in Petri dishes for three days at $4{ }^{\circ} \mathrm{C}$, seeds were transferred to $100-\mathrm{ml}$ pots with sand saturated with the Hoagland-Arnon $(\mathrm{H}-\mathrm{A})$ solution and grown in a plant growth cabinet (MLR-350H, "Sanyo", Japan) at $23^{\circ} / 19^{\circ} \mathrm{C}$ (day/night), $80 \%$ air relative humidity, at $120 \mu \mathrm{mol} \mathrm{m}^{-2} \mathrm{c}^{-1}$ PAR and 16-h photoperiod, as described by Shtratnikova et al. (2015). Preliminary experiments showed that etr1-1 grows more slowly at the early stages of development, thus mutants were planted four days earlier than Col-0. Plants received three $\mathrm{ml}$ of $\mathrm{H}$-A solution daily plus distilled water according to the pot weight to maintain sand humidity at $60 \%$ of its full capacity, as described earlier (Arkhipova et al., 2007). Two weeks after plant transfer to the growth chamber, we studied the sensitivity of Col-0 and etr1-1 plants to the addition of exogenous IAA and the sensitivity of Col-0 and tir 1 plants to treatment with 1-MCP. Growing the plants in the sand enabled separation of the roots from the substrate without their damage. To determine sensitivity of growth to exogenous hormone, freshly prepared solution of IAA was added each day to yield a final concentration of $5.7 \times 10^{-6} \mathrm{M}$, taking into account the volume of nutrient solution in the pot. Daily auxin treatment was necessary due to its rapid breakdown. The concentration was chosen in preliminary experiments as one resulting in the most pronounced response in the plants of wild type. Plants of Col- 0 and tir 1 were treated with 1-MCP as described by Vysotskaya et al. (2011). Solution of 1-MCP was prepared from precursor obtained as a gift from its producer (SmartFresh, AgroFresh Inc., USA). The precursor $(0.1 \mathrm{~g}$ per 1$)$ was diluted in $0.05 \%$ solution of the moistening agent Silwett L-77 (De Sangosse Ltd., Great Britain). Leaves of some of the plants were sprayed with the obtained solution in a dose enabling complete moistening of the leaves not later than five min after solution preparation, when gaseous 1-MCP was produced. The second group of plants (control) was treated with the moistening solution. 1-MCP treatment was repeated in four days when new ethylene receptors could form. Immediately after spraying, plants were covered with a polyethylene bag for limitation of gaseous1-MCP diffusion and placed into a dark closed box, since according to Sisler and Serek (1997), irreversible binding of 1-MCP with ethylene receptors takes place in the darkness. In the morning, plants were returned to the growth chamber. Four and seven days after the start of the treatments with auxin and 1-MCP, correspondingly, the mass of all roots of a plant and the length of the longest roots were measured $(n=20)$. IAA was introduced into the medium daily and could lead to a more rapid manifestation of the growth response. Plant were at the rosette stage when growth characteristics were measured, and the first signs of flowering appeared one week after conclusion of experiments.

\section{Results and Discussion}

The roots of etr1-1 mutants were longer than those of the wild type Col-0 (Fig. 1a). These results can be easily explained by ethylene inhibition of root elongation ( $\mathrm{Ru}-$ 


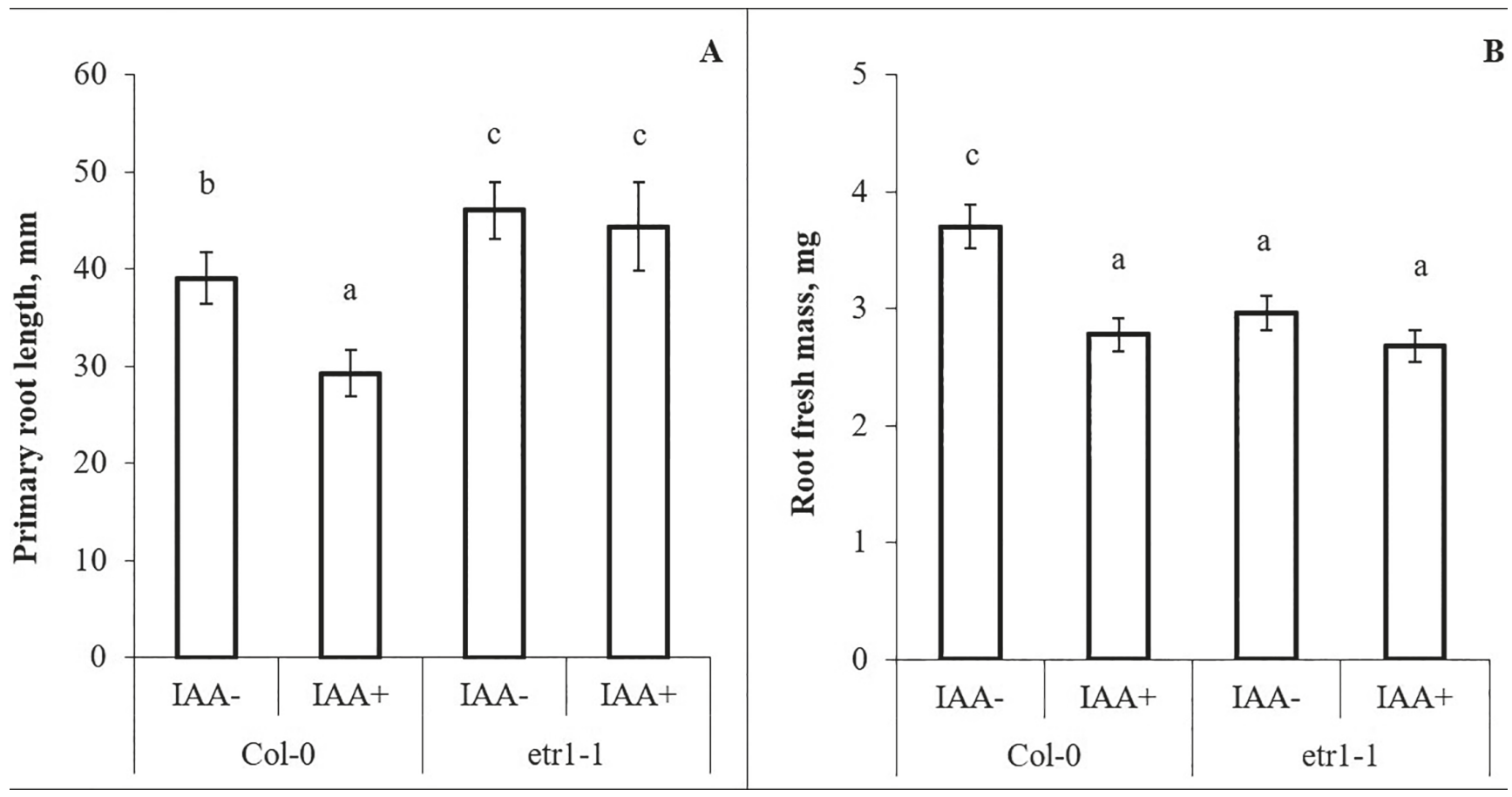

Fig. 1. Primary root length (A) and root fresh mass (B) of 18-days old ethylene insensitive Arabidopsis mutant etr1-1 and its parent ecotype Columbia (Col-0) 4 days after the start of IAA addition (IAA+) to the nutrient solution (to yield final concentration $5,7 \cdot 10^{-6} \mathrm{M}$ ). Statistically different means $(n=20)$ are indicated with different letters ( $L S D, p<0.05)$.

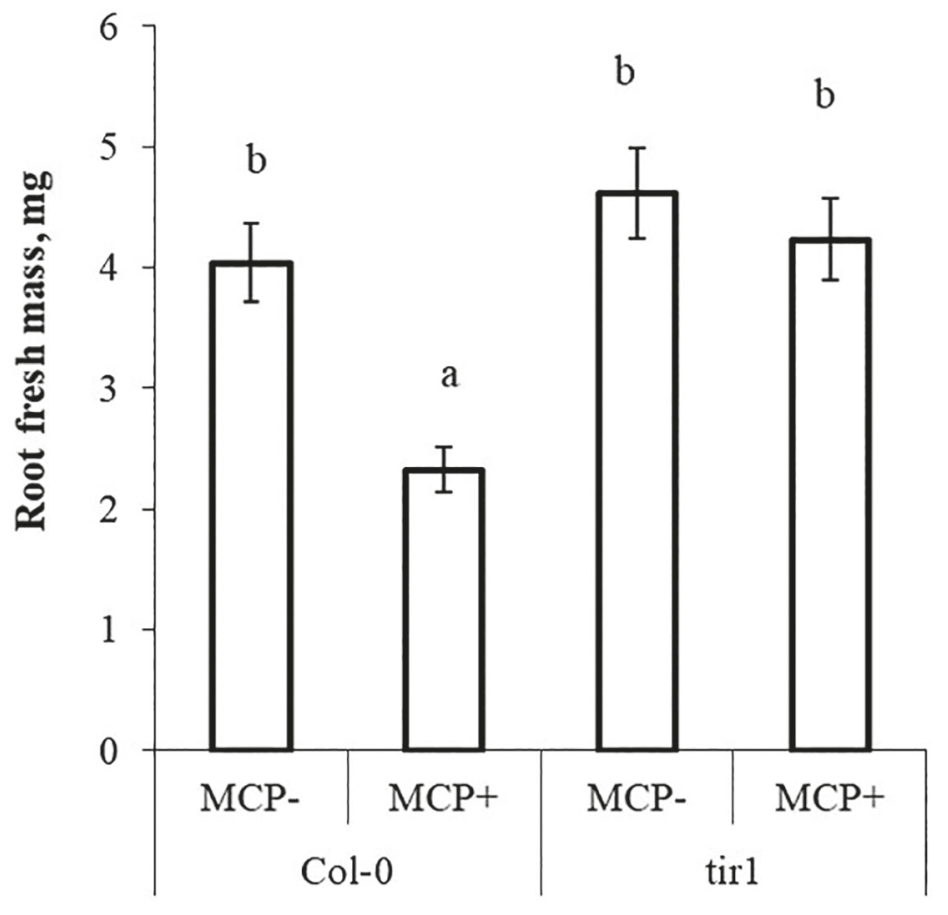

Fig. 2. Root fresh mass of 3-week old auxin insensitive Arabidopsis mutant tir1 and its parent ecotype Columbia (Col-0) 7 days after the start of shoot treatment with inhibitor of ethylene perception, 1-Methylcyclopropene (MCP+). Statistically different means $(n=20)$ are indicated with different letters $($ LSD, $p<0.05)$. zicka et al., 2007), while the loss of sensitivity to ethylene accelerates root elongation. Although root length in most publications is presented as a percentage and its absolute values are not provided, longer roots in etr 1 were detected by Contreras-Cornejo et al. (2015). Addition of IAA to the nutrient medium led to $20 \%$ root shortening in Col-0 plants as compared to the control (Fig. 1a).
In ethylene insensitive mutants the tendency of $5 \%$ root shortening compared to the control was statistically insignificant. Thus, plant roots that lost their sensitivity to ethylene were unable to respond to IAA in terms of their elongation. Previous experiments showed that the growth response of etr1-1 plants to synthetic auxin NAA was similar to root growth inhibition in plants of the 
wild type (Ruzicka et al., 2007). Since IAA crosses cell membranes less easily than NAA and needs transporters to a greater extent (Marchant et al., 1999), the capacity of NAA, but not IAA, to inhibit root elongation of etr $1 \mathrm{mu}-$ tants confirms the importance of the effect of ethylene on auxin transporters for inhibition of root elongation.

The roots of etr1-1 mutants were less heavy than those of Col-0 (Fig. 1b). These results are in accordance with the data obtained earlier in similar conditions (Korobova et al., 2016) and in the soil (Tholen et al., 2004). The results indicate that ethylene influences root elongation and mass accumulation in an opposite way. It makes roots shorter, but maintains accumulation of their mass. Thus, root response resembles to some extent the "triple response" detected in the stems and roots of dark-grown germinating seedlings, where ethylene induces their shortening and swelling (Gusman and Ecker, 1990).

Plant treatment with exogenous IAA induced a decrease in root mass of the plants of both tested genotypes. However, the effect was more obvious in the wild type plants: IAA treatment resulted in a 25 and $10 \%$ decrease in the root mass of Col- 0 and etr1-1, respectively, as compared to the corresponding control $(n=20$, the difference in the effect of IAA between the two genotypes is significant at $\mathrm{p} \leq 0.5$ ). Thus, the plants with the absence of sensitivity to ethylene retained the capacity to respond to exogenous IAA, manifested in the decrement in root biomass accumulation. Nevertheless, sensitivity to IAA in plants with a mutation of the ethylene receptor was lower than in the wild type plants. These results serve as evidence that IAA by itself (independently of ethylene) may influence root biomass accumulation. However, sensitivity to ethylene increases plants responsiveness to IAA.

Unlike genetic modification of sensitivity to ethylene, inhibition of ethylene perception with the 1-MCP did not significantly change the root length (data not shown). This is likely due to plant spraying with 1-MCP that mostly inactivated leaf, but not root receptors. Treatment of the plants of both genotypes with the inhibitor of ethylene perception (1-MCP) decreased the root mass. Chemical inactivation of ethylene receptors influences root mass accumulation in the same way as switching the receptors off in the mutant. Since, as indicated above, 1-MCP was likely to react mainly with the leaf receptors, the 1-MCP-induced decline in root mass could be a consequence of the effect of ethylene sensitivity on assimilate outflow from the shoots to the roots. Comparison of the growth response to 1-MCP treatment in Col-0 and tirl showed that mutation in the auxin receptor decreased the sensitivity of roots to inactivation of ethylene receptors: treatment with 1-MCP resulted in a $40 \%$ decline in the root mass of Col- 0 and only a $10 \%$ decline in tir 1 (the difference in the extent of root mass decline between the plants of the genotypes was significant at $\mathrm{p} \leq 0,01, \mathrm{n}=20$ ).

\section{Conclusion}

Our presented results suggest that the capacity of plants to sense ethylene plays an important role in the control of root length and root responsiveness to auxin. Mutations in the gene ETR1, which codes the ethylene receptor, contribute to root elongation in Arabidopsis and a significant decline in sensitivity of root elongation to exogenous IAA. These results differed from those obtained in experiments with the treatment of the tested mutant with synthetic auxin NAA, which easily diffuses across membranes and (unlike IAA) does need transporters (at least AUX1 (Ruzicka et al., 2007)). The difference between the responses of the ethylene-insensitive mutant to NAA and IAA suggests that the responses to IAA are dependent on ethylene sensitivity due to the effect of ethylene on auxin transporters, which is in accordance with the data of Ruzicka et al. (2007). The loss of sensitivity to ethylene influenced auxin's capacity to inhibit root mass accumulation to a lesser extent than root elongation. Nevertheless, the absence of sensitivity to ethylene in the etr1-1 mutant decreased responsiveness of root mass accumulation to the inhibiting action of IAA. In a similar way, the limitation of sensitivity to auxins in the tirl mutant decreased responsiveness of root biomass to 1-MCP-treatment. These data allow us to suggest an additive action of ethylene and auxins on the accumulation of root biomass and dependence of root elongation regulation by auxins on sensitivity to ethylene.

\section{References}

Alarcon, M. V., Lloret, P. G., Salguero, J. 2013. Auxin-ethylene interaction in transversal and longitudinal growth in maize primary root. Botany 91:680-685. https://doi. org/10.1139/cjb-2013-0133

Arkhipova, T. N., Prinsen, E., Veselov, S. Yu., Martinenko, E. V., Melentiev, A. I., Kudoyarova, G. R. 2007. Cytokinin producing bacteria enhances plant growth in drying soil. Plant and Soil 292:305-315. https://doi.org/10.1007/ s11104-007-9233-5

Contreras-Cornejo, H. A., López-Bucio, J. S., Méndez-Bravo, A., Macías-Rodríguez, L., Ramos-Vega, M., Guevara-García, Á. A., López-Bucio, J. 2015. Mitogen-activated protein kinase 6 and ethylene and auxin signaling pathways are involved in Arabidopsis root-system architecture alterations by Trichoderma atroviride. Molecular Plant-Microbe Interactions 28(6):701-710. https://doi.org/10.1094/MPMI-01-15-0005-R

Eliasson, L., Bertell, G., Bolander, E. 1989. Inhibitory action of auxin on root elongation not mediated by ethylene. Plant Physiology 91:310-314. https://doi.org/10.1104/pp.91.1.310

Guzman, P., Ecker, J.R. 1990. Exploiting the triple response of Arabidopsis to identity ethylene-related mutants. The Plant Cell 2:513-523. https://doi.org/10.1105/tpc.2.6.513

Ivanov, I. I. 2009. Endogenous auxins and branching of wheat roots gaining nutrients from isolated compartments. Russian Journal of Plant Physiology 56(2):219-223. https:// doi.org/10.1134/S1021443709020101

Korobova, A.V., Vysotskaya, L. B., Vasinskaya, A. N., Kuluev, B. R., Veselov, S. Yu., Kudoyarova, G. R. 2016. Dependence of root biomass accumulation on the content 
and metabolism of cytokinins in ethylene-insensitive plants. Russian Journal of Plant Physiology 63(5):597-603. https://doi.org/10.7868/S0015330316050079

Kudoyarova, G. R., Dodd, I. C., Veselov, D. S., Rothwell, S. A., Veselov, S.Y. 2015. Common and specific responses to availability of mineral nutrients and water. Journal of Experimental Botany 66:2133-2144. https://doi. org/10.1093/jxb/erv017

Marchant, A., Kargul, J., May, S. T., Muller, P., Delbarre, A., Perrot-Rechenmann, C., Bennett, M. J. 1999. AUX1 regulates root gravitropism in Arabidopsis by facilitating auxin uptake within root apical tissues. The EMBO Journal 18:2066-2073. https://doi.org/10.1093/emboj/18.8.2066

Muday, G. K., Rahman, A., Binder, B. M., Muday, G. K., Rahman, A., Binder, B. M. 2012. Auxin and ethylene: collaborators or competitors? Trends in Plant Science 17:181195. https://doi.org/10.1016/j.tplants.2012.02.001

Negi, S., Ivanchenko, M. G., Muday, G. K. 2008. Ethylene regulates lateral root formation and auxin transport in Arabidopsis thaliana. The Plant Journal 55:175-187. https://doi. org/10.1111/j.1365-313X. 2008.03495.x

Ruzicka, K., Ljung, K., Vanneste, S., Podhorska, R., Beeckman, T., Friml, J., Benkova, E. 2007. Ethylene regulates root growth through effects on auxin biosynthesis and transport-dependent auxin distribution. The Plant Cell 19:2197-2212. https://doi.org/10.1105/tpc.107.052126

Shtratnikova, V.Yu., Kudryakova, N. V., Kudoyarova, G. R., Korobova, A. V., Akhiyarova, G. R., Danilova, M. N., Kusnetsov, V. V., and Kulaeva, O. N. 2015. Effects of nitrate and ammonium on growth of Arabidopsis thaliana plants transformed with the ARR5::GUS construct and a role for cytokinins in suppression of disturbances in- duced by the presence of ammonium. Russian Journal of Plant Physiology 62:741-752. https://doi.org/10.7868/ S0015330315060159

Sisler, E. C., Serek, M. 1997. Inhibitors of ethylene responses in plants at the receptor level: recent developments. Physiologia Plantarum 100:577-582. https://doi. org/10.1111/j.1399-3054.1997.tb03063.x

Stepanova, A. N., Yun, J., Likhacheva, A. V., Alonso, J. M. 2007. Multilevel interactions between ethylene and auxin in Arabidopsis roots. The Plant Cell 19:2169-2185. https:// doi.org/10.1105/tpc.107.052068

Takahashi, H. 2013. Auxin biology in roots. Plant Root 7:49-64. https://doi.org/10.3117/plantroot.7.49

Tholen, D., Voesenek, L. A. C. J., Poorter, H. 2004. Ethylene insensitivity does not increase leaf area or relative growth rate in Arabidopsis, Nicotiana tabacum, and Petunia $x$ hybrid. Plant Physiology 134:1803-1812. https://doi. org/10.1104/pp.103.034389

Trapeznikov, V.K., Ivanov, I. I., Kudoyarova, G. R. 2003. Effect of heterogeneous distribution of nutrients on root growth, АБA content and drought resistance of wheat plants. Plant and Soil 252:207-214. https://doi. org/10.1023/A:1024734310214

Vysotskaya, L., Wilkinson, S., Davies, W., Arkhipova, T., Kudoyarova, G. 2011. The effect of competition from neighbours on stomatal conductance in lettuce and tomato plants. Plant Cell and Environment 34:729-737. https:// doi.org/10.1111/j.1365-3040.2011.02277.x

Woeste, K. E., Vogel, J. P., Kieber, J.J. 1999. Factors regulating ethylene biosynthesis in etiolated Arabidopsis thaliana seedlings. Physiologia Plantarum 105:478-484. https:// doi.org/10.1034/j.1399-3054.1999.105312.x 